\title{
An Inherited Arrhythmia Syndrome with Long QT, Sudden Death and Depolarization Disorder Due to an In-Frame Deletion in Exon 16 of the CACNA1C Gene
}

\author{
Muhammad Arshad Rafiq ${ }^{1,2}$, Tamara T Koopmann ${ }^{1}$, Laura A Zahavich ${ }^{3}$, Meena Fatah ${ }^{1}$ and Robert M Hamilton ${ }^{1 \star}$ \\ ${ }^{1}$ Physiology and Experimental Medicine, The Hospital for Sick Children and Research Institute, Toronto, Ontario, M5G 0A4, Canada \\ ${ }^{2}$ Department of Bio-Sciences, COMSATS Institute of Information Technology (CIIT) Park Road, Tarlai Kalan, Islamabad, 45550, Pakistan \\ ${ }^{3}$ The Hospital for Sick Children, Cardiology and Clinical Genetics, The Hospital for Sick Children and Research Institute, Toronto, Ontario, M5G 0A4, Canada
}

*Corresponding author: Robert M Hamilton, Physiology and Experimental Medicine, The Hospital for Sick Children and Research Institute, Toronto, Ontario, M5G 0A4 Canada, Tel: +4168136142; Fax: +4168137547; E-mail: robert.hamilton@sickkids.ca

Rec date: Feb 24, 2017; Acc date: Apr 10, 2017; Pub date: Apr 12, 2017

Copyright: (c) 2017 Rafiq MA, et al. This is an open-access article distributed under the terms of the creative commons attribution license, which permits unrestricted use, distribution, and reproduction in any medium, provided the original author and source are credited.

\begin{abstract}
Mutations of the gene encoding the L-type voltage gated calcium channel alpha-1C subunit (CACNA1C) underlie long QT phenotypes as part of Timothy syndrome. Milder phenotypes, as well as isolated cardiac phenotypes, including Brugada syndrome have been observed. To date, CACNA1C mutations have typically been missense mutations on limited number of sites that result in either gain of function (Timothy syndrome with a prolonged QT) or loss of function (short QT and/or Brugada pattern on ECG). We report a multiplex four-generation family with 3 individuals affected by QT prolongation, sudden cardiac death and conduction abnormalities, segregating with a novel heterozygous in-frame deletion mutation in exon 16 of CACNA1C resulting in a single amino acid deletion (p.Lys773del) discovered by using clinical gene panel testing at Invitae Corporation. Affected members are present in 3 consecutive generations (II, III and IV), and demonstrate only the cardiac rhythm phenotype segregating in an autosomal dominant fashion, with normal intellect, socialization and absence of syndactyly. The implicated in-frame deletion mutation of $C A C N A 1 C$ is absent from the exome aggregation consortium (ExAC) database, predicted to be disease causing by Mutation Taster, and removed an evolutionarily conserved lysine amino acid residue at position 773. Three-dimensional modelling demonstrated a marked effect of the mutation on the predicted protein structure.
\end{abstract}

Keywords: Long QT syndrome; Calcium channel; Mutation; Phenotype-genotype correlation

\section{Introduction}

Mutations of the gene encoding the L-type voltage gated calcium channel alpha-1C subunit (CACNA1C) were first identified in a syndrome of prolonged QT interval associated with hand and foot abnormalities and mental retardation or autism (Timothy syndrome) typically occurring de novo [1]. In subjects with mosaicism for the mutation, milder phenotypes can be observed and the disorder can be transmitted to offspring [2]. CACNA1C mutations have also been implicated in a small number of Brugada syndrome patients [3]. To date, CACNA1C mutations have typically been missense mutations on limited number of sites that result in either gain of function (Timothy syndrome with a prolonged QT) or loss of function (short QT and/or Brugada pattern on ECG).

\section{Clinical Description}

We report a multiplex four-generation family with 3 individuals affected by QT prolongation, sudden cardiac death and conduction abnormalities. The affected member in 3 generations (II, III and IV) demonstrated only the cardiac rhythm phenotype, with normal intellect and socialization and absence of syndactyly.

The family presented with a history of sudden cardiac death in the proband in his 30's (Figure 1). The proband's father was identified to have a prolonged QT interval, as well as atrial fibrillation, and was of
German ancestry. A daughter and son of the proband were evaluated. The son did not have a positive phenotype for disease, however the daughter had a low voltage QRS, prolonged QT interval and late potentials on signal-averaged ECG (performed for suspicion of phospholamban cardiomyopathy due to the low voltage ECG). There was no identified consanguinity in the family. The remaining family members depicted in the pedigree had no phenotype, based on clinical reports, as the features in this family were not typical for common forms of LQT syndrome.

\section{Methods and Results}

We performed an Arrhythmia Comprehensive Panel (Invitae Corporation) in the daughter. High quality DNA was extracted from 5 $\mathrm{ml}$ blood samples with Autopure LS (Qiagen) using the whole blood protocol and automated Puregene Chemistry at Genome Diagnostics Lab at the Department of Pediatric Laboratory Medicine, The Hospital for Sick Children, Toronto. High quality genomic DNA from the sample was sent to Invitae Corporation for clinical gene panel testing. The panel included screening of sequence changes and exonic duplications/deletions of $A B C C$ 9, ACTN2, AKAP9, ANK2, ANKRD1, CACNA1C, CACNA2D1, CACNB2, CALM1, CALM2, CALM3, CASQ2, CAV3, CTNNA3, DES, DSC2, DSG2, DSP, EMD, GPDIL, HCN4, JUP, KCND3, KCNE1, KCNE2, KCNE3, KCNE5, KCNH2, KCNJ2, KCNJ5, KCNJ*, KCNQ1, LDB3, LMNA, NKX2-5, PDLIM3, PKP2, PLN, PRKAG2, RANGRF, RBM20, RYR2, SCN10A, SCN1B, $S C N 2 B, S C N 3 B, S C N 4 B, S C N 5 A, S L M A P, S N T A 1, T G F B 3, T M E M 43$, TNNI3, TNNT2, TRDN, TRPM4 and TTN. 
Citation: Rafiq MA, Koopmann TT, Zahavich LA, Fatah M, Hamilton RM (2017) An Inherited Arrhythmia Syndrome with Long QT, Sudden Death and Depolarization Disorder Due to an In-Frame Deletion in Exon 16 of the CACNA1C Gene. Next Generat Sequenc \& Applic 4: 144. doi:10.4172/2469-9853.1000144

Page 2 of 4

Identified variants were characterized by their frequency in the Broad Exome Aggregation Consortium (ExAC, version) database where available [4]. In silico analysis was performed with Mutation Taster, an algorithm, which can be applied to insertions and deletions in addition to base pair substitutions. Three-dimensional modelling of the wild-type and mutated protein were performed with Iterative threading assembly refinement (I-TASSER) online software, a hierarchical approach to protein structure and function prediction. (http://zhanglab.ccmb.med.umich.edu/I-TASSER).

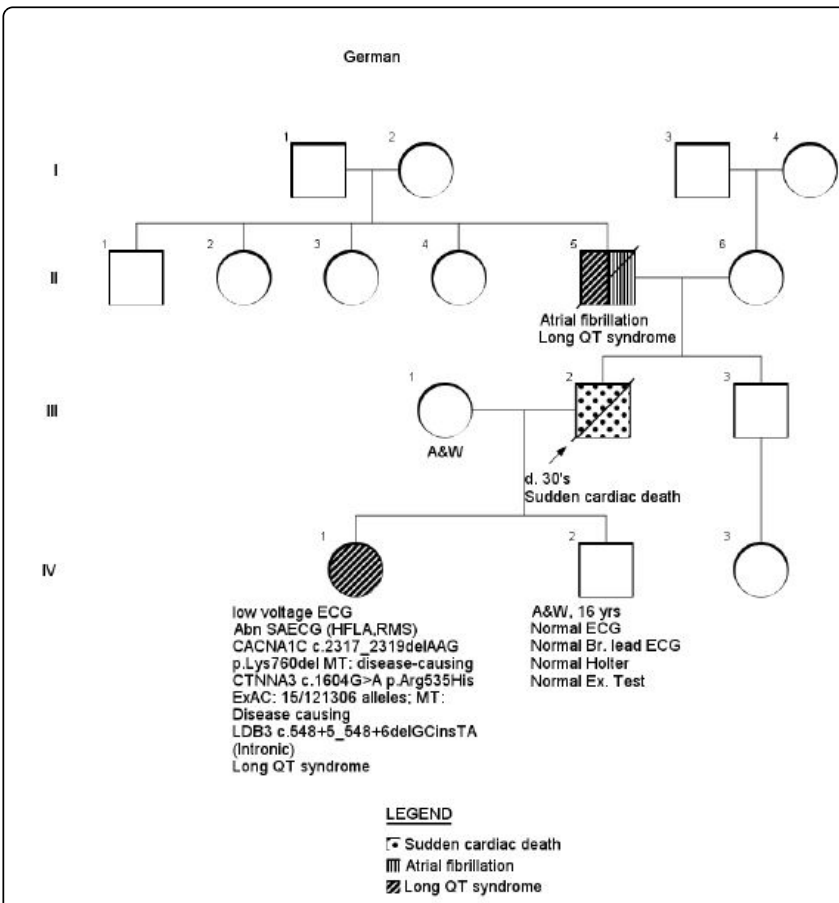

Figure 1: Four-generation pedigree of Family with three affected individuals (the proband III-2 died at the age of 30 , the proband's father II-5 had prolonged QT interval and atrial fibrillation and Proband's daughter IV-1 have a low voltage QRS, prolonged QT interval and late potentials on signal-averaged ECG.

Sequence analysis using the Invitae Arrhythmia Comprehensive Panel identified variants of uncertain significance in three genes: CACNA1C, CTNNA3 and $L D B 3$.

LDB3: A heterozygous variant in intron 5 of $L D B 3$ (c. $548+5 \_548+6$ delGCinsTA) was identified. This intronic variant is predicted to have minimal effect of gene splicing, and the typical phenotypes of LDB3 mutations (myofibrillar myopathy and/or dilated cardiomyopathy) were not present in this family.

CTNNA3: A heterozygous missense variant of CTNNA3 (c. $1604 G>A$; p.Arg534His), a recently identified gene underlying rare cases of arrhythmogenic right ventricular cardiomyopathy (ARVC), was identified. The variant is only moderately rare, being present in only 13/66713 alleles of the European sub-population in the ExAC database, and is not completely evolutionarily conserved. Mutation Taster predicts the variant to be "disease causing;" Polyphen-2 predicts it to be "moderately damaging," but SIFT predicts it to be "tolerated." However, given the frequency of the variant and difference in phenotype for this family from that predicted for this gene, we consider this variant as unlikely to be pathogenic [5-7].

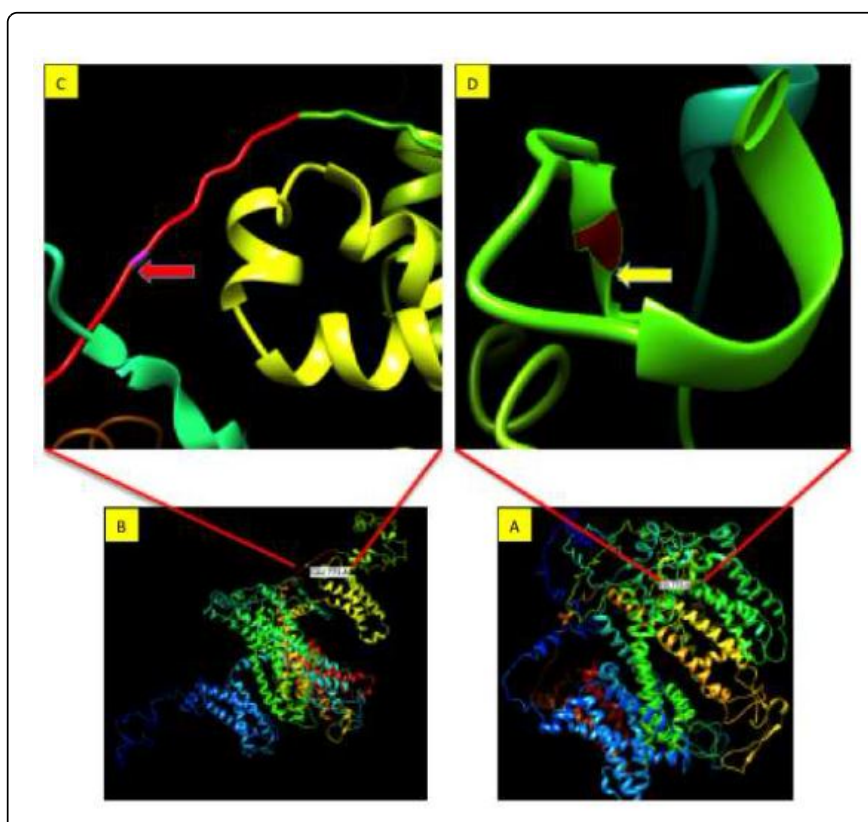

Figure 2: The predicted model of CACNAIC wild type (A) and mutant (B) proteins showing marked structural alteration. The panel C (unsprang) and D (coil) are captured by using Chimera tool to show deep structural difference due the presences or absences of Lys773.

CACNA1C: A heterozygous in-frame deletion in exon 16 of CACNA1C (c.2317_2319del AAG; p.Lys773del) was identified. The variant is novel based on its absence in 60706 unrelated individuals sequenced as part of various disease-specific and population genetic studies within the Exome Aggregation Consortium (ExAC, Broad Institute) database. Mutation taster (http://www.mutationtaster.org) classified the deletion as "disease-causing," as it deletes a lysine amino acid residue that is evolutionarily conserved across species. This lysine occurs at the ryanodine receptor interaction site of the II-III loop of $C A C N A 1 C$. The only other published mutation that has been identified in this linker region is Glu850del, in a poly ED motif associated with voltage dependent inactivation rather than the ryanodine receptor interaction site, and has been associated with loss of function resulting in early repolarization syndrome [8].

For both wild-type CACNA1C and the p.Lys773del mutant, 3dimensional structures were predicted using the I-TASSER program. Only 3-dimensional structures with high confidence scores (which can range from -5 to +2 ) are displayed (Figure 2). These scores were +1.05 for wild-type and +0.84 for the p.Lys773del mutant. For the p.Lys773del mutant, the missing lysine in the middle of the protein is predicted to result in a major structural alteration, with the N-terminal structure becoming 'unsprung' from the remainder of the protein. The predicted secondary structure of wild type (helix: $49.1 \%$, Sheets: $1.3 \%$, turns: $15 \%$ and coils: $32.7 \%$ ) and mutant protein (helix: $49.4 \%$, Sheets: $1.3 \%$, turns: $13 \%$ and coils: $33.9 \%$ ) also showed a difference in helix, turns and coils formations (Figure 3). 
Citation: $\quad$ Rafiq MA, Koopmann TT, Zahavich LA, Fatah M, Hamilton RM (2017) An Inherited Arrhythmia Syndrome with Long QT, Sudden Death and Depolarization Disorder Due to an In-Frame Deletion in Exon 16 of the CACNA1C Gene. Next Generat Sequenc \& Applic 4: 144. doi:10.4172/2469-9853.1000144

Page 3 of 4

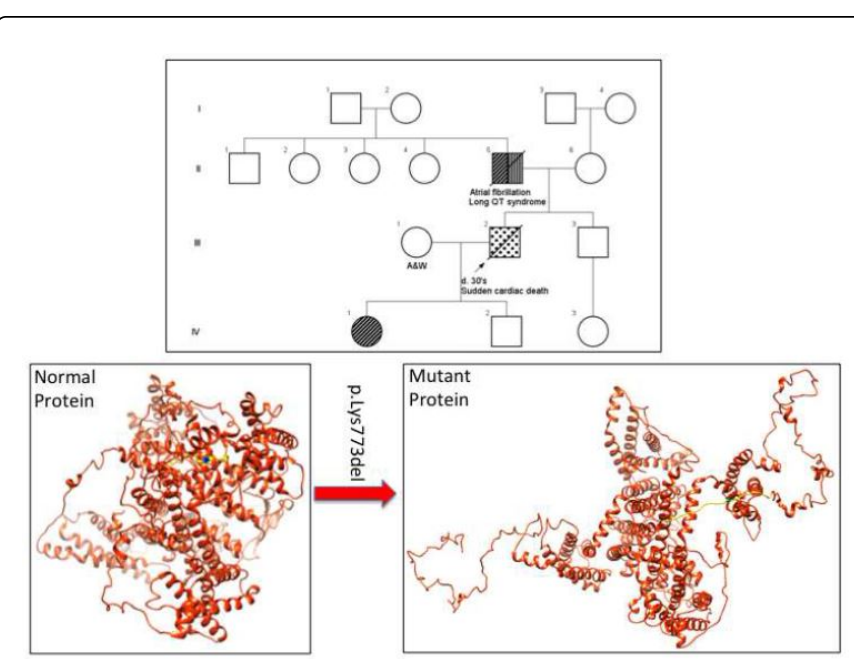

Figure 3: Secondary structure of wild type and mutant protein.

Of the three variants, the CACNA1C p.Lys773del variant is considered the culprit mutation, as it is very rare (in keeping with the rareness of long QT syndrome) is predicted by in silico analysis to be deleterious, and has major effect on the predicted structure of the $C A C N A 1 C$ protein. In addition, a p.CACNA1C Lys773del variant is also reported in ClinVar (NM_000719.6) by GeneDx (which includes CACNA1C sequencing on its long QT testing panel) as a variant of uncertain significance. Two additional unrelated probands with a similar CACNA1C exon16 3-base pair deletion (c.2314_2316delGAG) have also been reported [9]. In contrast, the $L D B 3$ variant has minimal effect on protein expression and the CTNNA3 variant is likely too common to be causative of a rare disease phenotype. Neither $L D B 3$ nor $C T N N A 3$ are known to cause repolarization disorder.

\section{Discussion}

Long QT syndromes are major contributor to inherited arrhythmia syndromes and results in sudden cardiac death. Although loss of function mutations of two cardiac potassium channels and gain of function mutations of the cardiac sodium channel underlie the bulk of long QT syndrome patients/families (LQT 1, 2 and 3), mutations of multiple ion channels or channel-interacting proteins (LQT 4-15) account for an additional $\sim 5 \%$. Some also have additional cardiac or extra-cardiac features beyond QT prolongation, resulting in unique cardiac phenotypes. Reversal or modification of the gain/loss effects by mutations of some genes may also result in opposing phenotypes such as Brugada syndrome, or overlapping phenotypes.

The L-type voltage gated calcium channel, and in particular its poreforming a1C subunit, is vital to cardiac muscle, linking the action potential upstroke in triggering of calcium release from the sarcoplasmic reticulum, thus initiating cardiac contraction. These channels also function in smooth muscle, neuronal and endocrine tissues, and are involved in regulation of transcription. Mutation of the a1C subunit (CACNA1C G406R) were first identified in a syndrome of prolonged QT interval, hand and foot abnormalities and mental retardation or autism (Timothy syndrome) typically occurring de novo. An atypical form of Timothy syndrome, type 2 (TS2), has also been described due to de novo G402S in exon 8 of the CACNA1C gene, both with and without mosaicism and demonstrates isolated cardiac arrhythmias $[10,11]$. Among 540 unrelated subjects with LQT syndrome, novel or very rare $C A C N A 1 C$ variants were identified in six (de novo in 3, inherited along with phenotype in 3) [12]. A threegeneration family with five members variably affected with LQT, hypertrophic cardiomyopathy and sudden cardiac death demonstrated segregation of a novel CACNA1C R518C mutation [13]. Among 82 victims of sudden unexpected death in the young, a novel $C A C N A 1 C$ $N 2091 S$ gain of function mutation has also been identified [14].

The marked alteration caused in the predicted structure of CACNA1C by the deletion of Lysine in amino acid position 773 is likely contributing to gain of function, given the long QT phenotype prevalent among the affected subjects. This gain of function may be mediated by increased calcium channel current amplitude, a negative shift in voltage-dependence of activation, slowed voltage-dependent inactivation, positive shift in voltage-dependence of inactivation or reduced steady-state inactivation, as previously described among missense mutations of $C A C N A 1 C$ underlying long QT syndrome [12].

\section{Conclusion}

We report a p.Lys773del mutation in the CACNA1C (L-type calcium channel alc subunit) gene identified in the youngest member of a human pedigree presenting with prolonged QT or sudden cardiac death in 3 individuals across 3 generations. The mutation is novel, predicted to be pathogenic, and alters the predicted protein structure of the calcium channel alpha subunit protein.

\section{Acknowledgment}

The authors thank the individuals/family who participated in this study. Funding sources include the Canadian Institutes of Health Research, The Carter Heart Rhythm Fellowship, The Caitlin Morris Memorial Fund, The Alex Corrance Memorial Fund and the Ted Rogers Centre for Heart Research.

\section{References}

1. Splawski I, Timothy KW, Sharpe LM, Decher N, Kumar P, et al. (2004) $\mathrm{Ca}(\mathrm{V}) 1.2$ calcium channel dysfunction causes a multisystem disorder including arrhythmia and autism. Cell 119: 19-31.

2. Etheridge SP, Bowles NE, Arrington CB, Pilcher T, Rope A, et al. (2011) Somatic mosaicism contributes to phenotypic variation in Timothy syndrome. Am J Med Genet A 155A: 2578-2583.

3. Antzelevitch C, Pollevick GD, Cordeiro JM, Casis O, Sanguinetti MC, et al. (2007) Loss-of-function mutations in the cardiac calcium channel underlie a new clinical entity characterized by ST-segment elevation, short QT intervals, and sudden cardiac death. Circulation 115: 442-449.

4. Lek M, Karczewski KJ, Minikel EV, Samocha KE, Banks E, et al. (2016) Analysis of protein-coding genetic variation in 60,706 humans. Nature 536: 285-291.

5. Schwarz JM, Cooper DN, Schuelke M, Seelow D (2014) MutationTaster2: Mutation prediction for the deep sequencing age Nat Methods 11: 361-362.

6. Adzhubei IA, Schmidt S, Peshkin L, Ramensky VE, Gerasimova A, et al. (2010) A method and server for predicting damaging missense mutations. Nat Methods 7: 248-249.

7. Ng PC, Henikoff S (2003) SIFT: Predicting amino acid changes that affect protein function. Nucleic Acids Res 31: 3812-3814.

8. Turner RW, Anderson D, Zamponi GW (2011) Signaling complexes of voltage-gated calcium channels. Channels 5: 440-448.

9. Marshall AM, Kugler JD, Mill L, Houston K, Starr L, et al. (2015) CACNA1C exon 16 variant in patients with polymorphic ventricular tachycardia. Midwest Pediatric Cardiology Society 2015 annual Meeting. 
Citation: $\quad$ Rafiq MA, Koopmann TT, Zahavich LA, Fatah M, Hamilton RM (2017) An Inherited Arrhythmia Syndrome with Long QT, Sudden Death and Depolarization Disorder Due to an In-Frame Deletion in Exon 16 of the CACNA1C Gene. Next Generat Sequenc \& Applic 4: 144. doi:10.4172/2469-9853.1000144

Page 4 of 4

10. Splawski I, Timothy KW, Decher N, Kumar P, Sachse FB, et al. (2005) Severe arrhythmia disorder caused by cardiac L-type calcium channel mutations. Proc Natl Acad Sci U S A 102: 8089-8096.

11. Hiippala A, Tallila J, Myllykangas S, Koskenvuo JW, Alastalo TP (2015) Expanding the phenotype of Timothy syndrome type 2: An adolescent with ventricular fibrillation but normal development. Am J Med Genet A 167A: 629-634.

12. Wemhöner K, Friedrich C, Stallmeyer B, Coffey AJ, Grace A, et al. (2015) Gain-of-function mutations in the calcium channel CACNA1C (Cav1.2) cause non-syndromic long-QT but not Timothy syndrome. J Mol Cell Cardiol 80: 186-195.
13. Boczek NJ, Ye D, Jin F, Tester DJ, Huseby A, et al. (2015) Identification and functional characterization of a novel CACNA1C-mediated cardiac disorder characterized by prolonged QT intervals with hypertrophic cardiomyopathy, congenital heart defects and sudden cardiac death. Circ Arrhythm Electrophysiol 8: 1122-1132.

14. Sutphin BS, Boczek NJ, Barajas-Martínez H, Hu D, Ye D, et al. (2016) Molecular and functional characterization of rare CACNA1C variants in sudden unexplained death in the young. Congenit Heart Dis 11: 683-692. 